\title{
HF waves heating ionosphere F-layer
}

\author{
FANG HanXian ${ }^{1,2}$, WANG SiCheng ${ }^{1,2^{*}} \&$ SHENG Zheng ${ }^{1}$ \\ ${ }^{1}$ Institute of Meteorology, PLA University of Science and Technology, Nanjing 211101, China; \\ ${ }^{2}$ State Key Laboratory of Space Weather, Chinese Academy of Sciences, Beijing 100190, China
}

Received January 20, 2012; accepted April 23, 2012; published online September 6, 2012

\begin{abstract}
To predict and evaluate the effects of ionospheric modification quantitatively, the electron temperature and density perturbations are analyzed at different heating conditions, based on a developed model including momentum equation, continuity equation and energy equation. The results show that (1) powerful HF radio waves can cause the electron temperature to increase markedly, and the largest perturbation is near the reflection layer, but the electron density decreases at this area; (2) the lower neutral gas density in the ionosphere, the larger disturbance of the electron temperature and density; the higher radio frequency, the lower increment of electron temperature; and the higher radio power, the larger disturbance of electron temperature and density are caused, but there are no linear relationships between the radio power and disturbance amplitude; (3) the perturbations at nighttime are larger than at daytime when heated by HF radio waves with the same powers; the electron temperature perturbations in the solar minimum are larger than that in solar maximum; and the electron temperature perturbations in spring and autumn are larger than in winter and summer; (4) compared with the low-latitude, the mid-latitude has the smaller perturbations, the maximum electron temperature perturbation is $\sim 30 \%$ (at daytime of winter), and the density is $\sim 5 \%$.
\end{abstract}

powerful HF waves, ionospheric heating, simulation

Citation: $\quad$ Fang H X, Wang S C, Sheng Z. HF waves heating ionosphere F-layer. Chin Sci Bull, 2012, 57: 4036-4042, doi: 10.1007/s11434-012-5408-4

The ionosphere is the main carrier and channels of radio waves; the conditions of ionosphere can strongly affect the quality of the satellite-ground and long-distance groundground radio wave communications. As for the ionosphere, it is a very complex system, and is not only influenced by the Sun and magnetosphere-driven processes [1-5], but also influenced by dynamical atmospheric processes. For example, lightning can heat ionosphere to result in modification of the electron temperature and number density [6,7]; earthquakes may produce pre-earthquake ionospheric anomalies [8-12]; typhoon or hurricanes can cause ionospheric TEC increase and foF2 to rise [13]; atmospheric waves (planetary, gravity, tidal and infrasonic waves) coming from below can significantly affect the lower ionosphere, both directly and indirectly (e.g. through major stratospheric warmings) [14-16].

Except for these natural ionospheric disturbances, many artificial methods can also cause the ionosphere to be dis-

\footnotetext{
*Corresponding author (email: ionosphere.nj@gmail.com)
}

turbed [17-19]. Since 'Luxemburg effect' was found in 1933, it has been known for many years that the ambient properties of the Earth's ionosphere can be modified by the absorption of powerful HF waves launched by groundbased transmitters. With the merit of convenience, swift and notable heating effect and being friendly to environment, ground-based transmitters became one of the major ionospheric modification means. Especially after 1970s, USA, Soviet Russia and some European countries have established much experimental equipment. HAARP facility in Alaska now owns the largest power and antenna gain (the effective power could reach up to $1 \mathrm{GW}$ ), and can transmit strong electromagnetic energy flux to efficiently modify the ionosphere.

Powerful HF radio waves can modify the local electron temperature and density, and induce the change of plasma conductivity, dielectric constant and other plasma parameters. Heating the ionosphere not only has scientific research values, but also possesses practical objectives. For example, heating lower ionosphere $(50-100 \mathrm{~km})$ can cause ionization 
that forming 'ionospheric mirror' to reflect some particular electromagnetic waves; heating lower ionosphere by amplitude-modulated HF waves can efficiently produce radiation sources of ELF/VLF waves [20]; heating the upper ionosphere $(150-500 \mathrm{~km})$ could modify the electron density, form focusing and defocusing area that badly change or even disrupt the communication channel, and can also cause parametric instabilities and self-focusing instabilities to form various-scale field-aligned irregularities.

Because heating experiment is extremely expensive and heating is local and short-lived, all kinds of numerical models were developed with the increasing knowledge of the ionosphere physics [21-24]. Huang et al. [25] developed a model based on the achievement of other researchers, and the model is in accord with some experiment results. In this paper, we use this model to predict and evaluate the effects of ionospheric modification quantitatively, aiming to provide some guidance to the future experiments.

\section{Physics of large-scale modifications}

Under the well-justified assumption of ambipolar diffusion, the macroscopic dynamics of the $F$ region plasma can thus be described by following equations:

Momentum equation

$$
N_{e} V_{e}=-D\left[\frac{\partial}{\partial s} N_{e} k\left(T_{e}+T_{i}\right)+\sum_{\alpha} N_{\alpha} m_{\alpha} g \sin \theta\right]+N_{e} V_{n} \cos \theta
$$

Continuity equation

$$
\frac{\partial N_{e}}{\partial t}=Q_{0}-\beta N_{e}-\frac{\partial\left(N_{e} V_{e}\right)}{\partial s}
$$

Energy equation

$$
\begin{gathered}
\frac{3}{2} k\left[N_{e} \frac{\partial T_{e}}{\partial t}+N_{e} V_{e} \frac{\partial T_{e}}{\partial s}\right]+k N_{e} T_{e} \frac{\partial V_{e}}{\partial s} \\
=\frac{\partial}{\partial s}\left(K_{e} \frac{\partial T_{e}}{\partial s}\right)+S_{Q}+S_{0}-L_{e},
\end{gathered}
$$

with $s$ the coordinate along the magnetic field; $N_{e}$ the electron density; $T_{e}$ and $T_{i}$ the electron and ion temperature, respectively; $V_{e}$ the field-aligned flow velocity; $V_{n}$ the neutral wind velocity in the north to south direction; $N_{\alpha}$ the ion density (mainly consider $\mathrm{O}^{+}, \mathrm{NO}^{+}$and $\mathrm{O}_{2}^{+}$); $m_{\alpha}$ the ion mass; $k$ the Boltzmann constant; $g$ the acceleration due to gravity; $\theta$ the magnetic field dip angle; and $D, \beta, K_{e}$ and $L_{e}$ the coefficients of density diffusion, recombination, thermal conduction and cooling, the conduction coefficient has an approximately $T_{e}^{5 / 2}$ behavior; $S_{Q}$ the heating source due to radio waves; $Q_{0}$ and $S_{0}$ the local ionization source and energy absorption source in natural conditions respectively.

Heating can result from direct Ohmic absorption by the bulk plasma electrons as well as from the slowing down of superthermal electrons accelerated by short-scale length waves excited by the HF pump. For simplicity, energy absorption is limited to a Gaussian centered at the reflection height [24,26,27], and the energy absorption at the reflection height can be written as [22]

$$
\begin{aligned}
S_{Q, \max } & =\frac{\varepsilon_{0}}{2}\left(\frac{f_{p}^{2} v_{e}}{f_{0}^{2}}\right) E_{z_{m}}^{2}+16\left(\frac{n_{e} T_{e}}{\omega}\right) v_{e}^{2}\left(\frac{E_{z_{m}}}{E_{t}}\right)^{4} \\
& =A \frac{\varepsilon_{0}}{2}\left(\frac{f_{p}^{2} v_{e}}{f_{0}^{2}}\right) E_{z_{m}}^{2}\left(\mathrm{~J} \mathrm{~m}^{-3} \mathrm{~s}^{-1}\right),
\end{aligned}
$$

where $A$ is a constant, and it generally equals to 10 according to the experiments; $\varepsilon_{0}$ the dielectric constant; $f_{p}$ the local plasma frequency; $f_{0}$ the radio frequency; $v_{e}$ the electron collision frequency, mainly include electron-neutral $\left(v_{e n}\right)$ and electron-ion $\left(v_{e i}\right)$ collision; $z_{m}$ the altitude of reflection point; $E$ the electric field intensity; $E_{t}$ the abnormal absorption excitation threshold. $v_{e n}$ and $v_{e i}$ can derive from empirical equations as follows [28]:

$$
\begin{gathered}
v_{e n}=9.32 \times 10^{-12} N_{\mathrm{N}_{2}}\left(1-3.44 \times 10^{-5} T_{e}\right)+1.21 \times 10^{-10} N_{\mathrm{O}_{2}} \\
\times\left(1+2.15 \times 10^{-12} T_{e}^{0.5}\right) T_{e}+5.49 \times 10^{-10} N_{\mathrm{O}} T_{e}^{0.5}, \\
v_{e i}=54 \frac{N_{e}}{T_{e}^{3 / 2}} .
\end{gathered}
$$

The absorption energy at arbitrary ionospheric altitude $z$ $(\mathrm{km})$ is

$$
S_{Q}(z)=S_{Q, \max } \mathrm{e}^{-\left(z-z_{m}\right)^{2} / \sigma^{2}},
$$

where $\sigma$ the half-width of Gaussian, generally assumes to 20 $\mathrm{km}$. The electric field intensity of HF waves as a function of height can approximately be written as [29]

$$
E(z)=\frac{\sqrt{30 E R P}}{z} .
$$

Here $E R P$ is the transmitter effective radiation power.

The loss processes mainly include (1) elastic collisions with positive ions and neutral particles, (2) rotational excitation of $\mathrm{O}_{2}$ and $\mathrm{N}_{2}$, (3) vibrational excitation of $\mathrm{O}_{2}$ and $\mathrm{N}_{2}$, (4) electronic excitation of $\mathrm{O}$, (5) fine structure excitation of O. Ref. [30] provides the detailed expressions.

\section{Computational arithmetic}

We use Crank-Nicolson method [31] to solve the equation (3); this method was developed by John Crank and Phyllis Nicolson in the mid 20th century. In numerical analysis, the Crank-Nicolson method is a finite difference method used for numerically solving the heat equation and similar partial 
differential equations. It is a second-order method in time, implicit in time, and is numerically stable.

Assuming the altitude $z$ ranges from $z_{\min }$ to $z_{\max }$ and the time $t$ from 0 to $T$. Divide $[0, T]$ into $N$ equally spaced intervals at $t$ values indexed by $l=0,1, \ldots N$, and $\left[z_{\min }, z_{\max }\right]$ into $I$ intervals at $z$ values indexed by $i=0,1, \ldots I$. The length of these intervals is $\Delta t$ in the time direction and $\Delta z$ in the $z$ direction. We seek an approximation to the true values of $T_{e}$ at the $(N+1) \times(I+1)$ grid points.

Focusing on an arbitrary internal grid point $(i, l+1)$, one could approximate the partial derivatives at that point by

$$
\begin{aligned}
& \frac{\partial T_{e}}{\partial t}=\frac{T_{e, i}^{l+1}-T_{e, i}^{l}}{\Delta t}, \\
& \frac{\partial T_{e}}{\partial z}=\frac{1}{2}\left[\frac{T_{e, i+1}^{l}-T_{e, i-1}^{l}}{2 \Delta z}+\frac{T_{e, i+1}^{l+1}-T_{e, i-1}^{l+1}}{2 \Delta z}\right], \\
& \frac{\partial^{2} T_{e}}{\partial z^{2}}=\frac{1}{2}\left[\begin{array}{c}
\frac{\left(T_{e, i+1}^{l}-T_{e, i}^{l}\right)-\left(T_{e, i}^{l}-T_{e, i-1}^{l}\right)}{(\Delta z)^{2}} \\
+\frac{\left(T_{e, i+1}^{l+1}-T_{e, i}^{l+1}\right)-\left(T_{e, i}^{l+1}-T_{e, i-1}^{l+1}\right)}{(\Delta z)^{2}}
\end{array}\right] \text {. }
\end{aligned}
$$

Written in matrix form, eq. (3) provides the interior equations of

$$
\left[\begin{array}{ccccc}
a_{1} & b_{1} & 0 & \ldots & 0 \\
c_{1} & a_{2} & b_{2} & \ddots & 0 \\
0 & c_{2} & a_{3} & \ddots & 0 \\
0 & \ddots & \ddots & \ddots & b_{n-1} \\
0 & \ldots & 0 & c_{n-1} & a_{n}
\end{array}\right]\left[\begin{array}{c}
T_{e, 1}^{l+1} \\
T_{e, 2}^{l+1} \\
T_{e, 3}^{++1} \\
\vdots \\
T_{e, n}^{l+1}
\end{array}\right]=\left[\begin{array}{c}
D_{1} \\
D_{2} \\
D_{3} \\
\vdots \\
D_{n}
\end{array}\right] .
$$

This sort of system is efficiently solved by Gaussian elimination. It requires just $8 I$ floating-point operations to determine the unknown $T_{e}^{l+1}$ of all heights, and then update the parameters which are related to the electron temperature, and finally use eq. (2) to calculate the electron

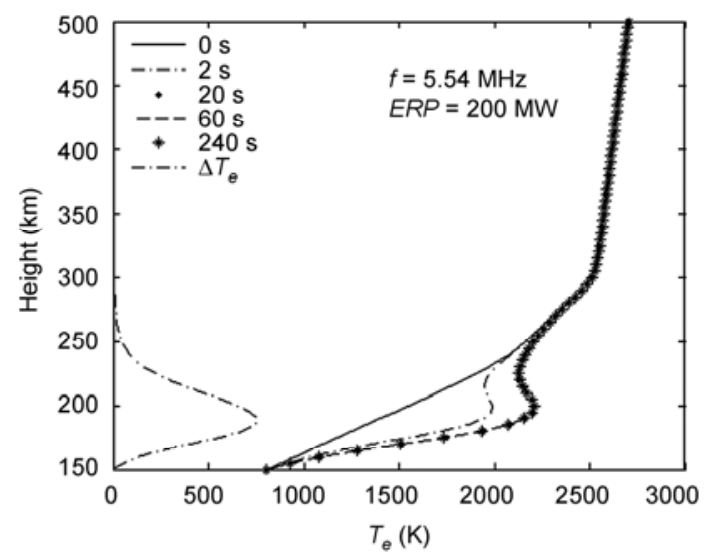

density of all altitudes.

\section{Simulation results}

The background parameters of ionosphere are obtained from the model IRI2007, and neutral density profiles are from the model NRLMSISE-00. The boundary conditions are the same as that in [24]; that is, the grads of electron temperature and density keep invariability at the upper boundary, and the electron temperature and density remain constant over the course of the calculation at the lower boundary.

The ionospheric altitude ranges from 150 to $500 \mathrm{~km}$, and heating at the midday of the winter in 2011 at Nanjing (geographic coordinate: $32^{\circ} \mathrm{N}, 118.5^{\circ} \mathrm{E}$, dip angle: $47.3^{\circ}$ ). Under these conditions $\mathrm{h}_{\mathrm{m}} \mathrm{F}_{2}$ is $275 \mathrm{~km}$, and foF $\mathrm{F}_{2}$ is $11.5 \mathrm{MHz}$. The transmitter frequency is $5.54 \mathrm{MHz}$, ERP is $200 \mathrm{MW}$, heating duration $4 \mathrm{~min}$, and the wave reflection height 195 $\mathrm{km}$. The electron temperature and density disturbance profiles are displayed in Figure 1. The electron temperature increases evidently just after heating $2 \mathrm{~s}$, and it is close to saturation when heating $10 \mathrm{~s}$. The maximum disturbance is $\sim 750 \mathrm{~K}$, near the reflection area. The electron density decreases at reflection area, and it mainly depends on the grads of electron temperature which makes the plasma diffuse along the magnetic field. The saturation time of the electron density is longer than that of the electron temperature, and the electron density is still unsaturated after heating $240 \mathrm{~s}$.

Figure 2 shows the disturbance profile of electron temperature and density when altering the background neutral density. We alter the neutral gas density to $0.8 N, 1.0 N, 1.2 N$ $(N$ is the original neutral density) respectively. Under $0.8 N$ conditions, the disturbance amplitude is the largest, while $1.2 \mathrm{~N}$ is the least. Therefore we can conclude that the lower background neutral density, the larger disturbance of electron temperature and density are caused.

To compare the disturbance amplitude of different heating

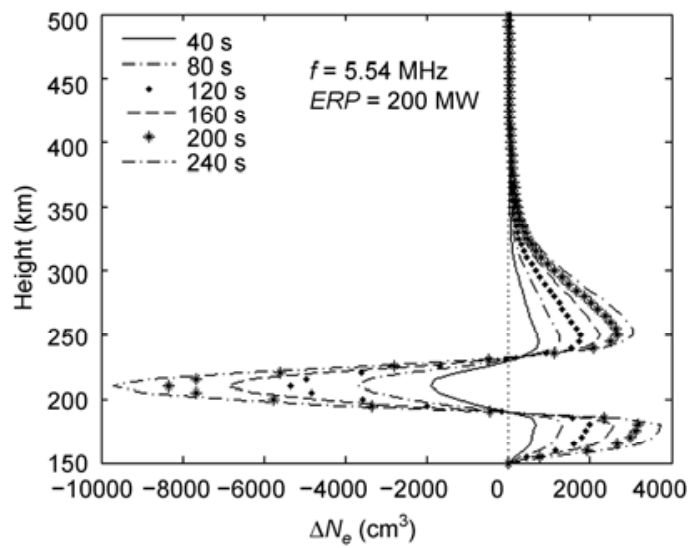

Figure 1 Profiles of electron temperature and density after disturbed. 

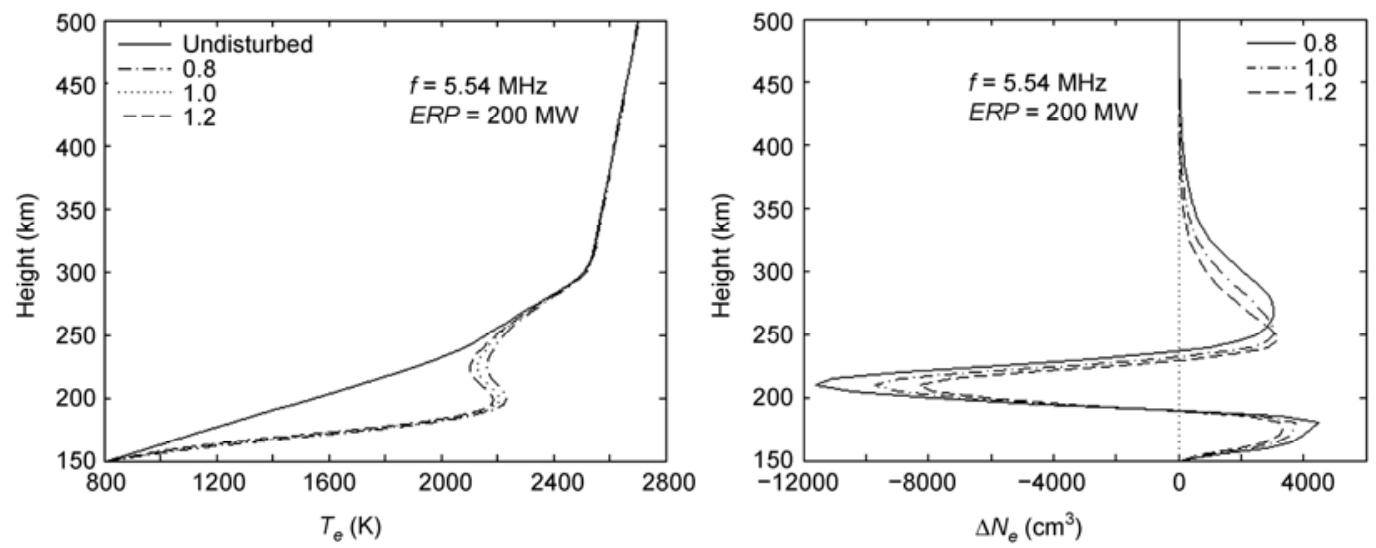

Figure 2 Profiles of electron temperature and density when changing neutral background density.

conditions, we design four sets of experiments as follows.

Simulation 1 Choosing the different transmitter frequencies and powers to heat the ionosphere $4 \mathrm{~min}$, the disturbance profiles are showed in Figure 3. Under the same power conditions, the larger radio frequency, the higher reflection altitude, and the lower disturbance of electron temperature. The higher radio power, the larger disturbance of electron temperature and density if the frequencies are the same, but there are no linear relationships between the disturbance amplitude and radio power.

Simulation 2 Choosing the different moments to heat the ionosphere. The transmitter frequency is set to near the foF 2 , radio power is $200 \mathrm{MW}$, and the results are shown in Figure 4. The electron temperature increases at all heights, the maximum amplitude at daytime is $\sim 20 \%$, and the nighttime can reach up to $\sim 300 \%$; while the electron density
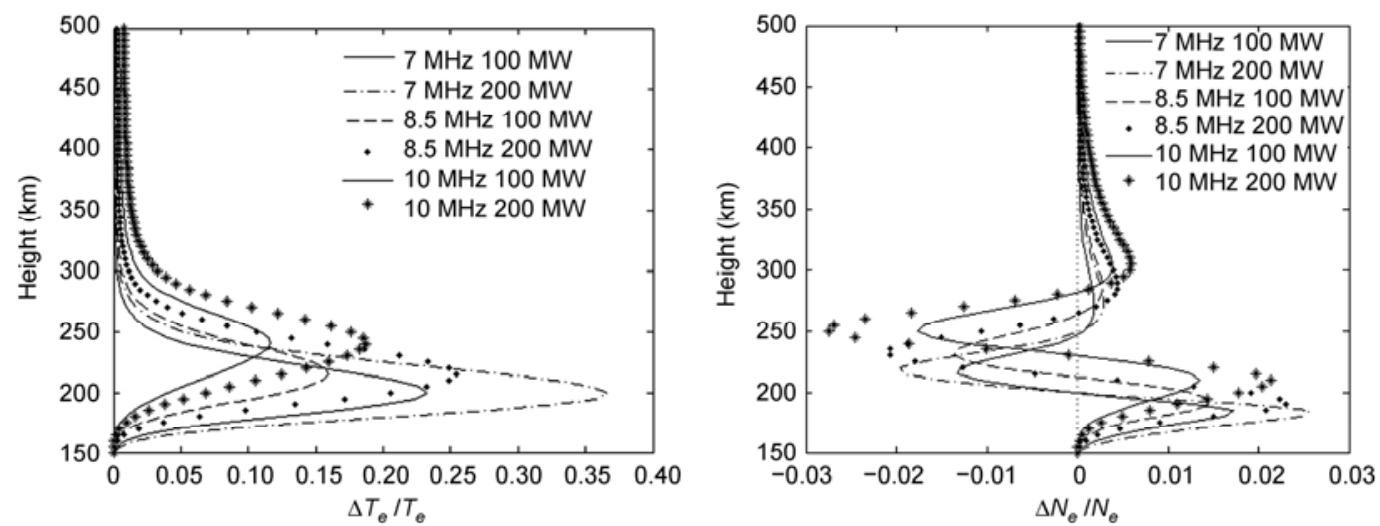

Figure 3 Profiles of electron temperature and density under the simulation 1 conditions.
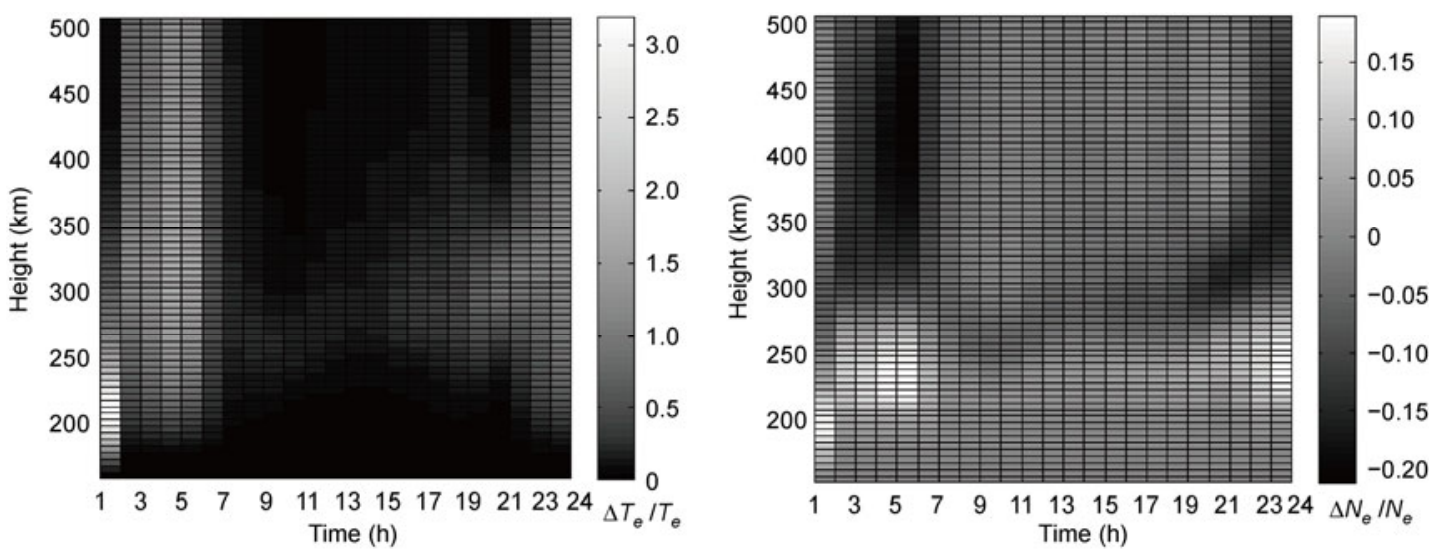

Figure 4 Profiles of electron temperature and density under the simulation 2 conditions. 
at daytime is $\sim 4 \%$, and the nighttime is $\sim 20 \%$. By comparison, the disturbance at nighttime is larger than at daytime when heated by HF radio waves. This is in accordance with the experiments [32,33], and many experiments are conducted at nighttime. Possible reason is that the nighttime has lower plasma parameters than the daytime, when the radio waves with the same power interact with the ionosphere, more energy could transport to the electrons and induce larger ionospheric disturbance at nighttime.

Simulation 3 Choosing the different latitudes to heat the ionosphere. The latitude ranges from $0^{\circ} \mathrm{N}$ to $90^{\circ} \mathrm{N}\left(10^{\circ}\right.$ spacing), the ERP of transmitter is $200 \mathrm{MW}$, and radio frequency is $\sim \mathrm{foF}_{2}$, heating duration is $4 \mathrm{~min}$. At low latitude, the electron temperature disturbance amplitude could reach up to $\sim 80 \%$, and electron density $\sim 20 \%$. While compared with the low latitude, the mid-latitude has the smaller disturbance, the electron temperature is $\sim 30 \%$, and the electron density is $\sim 5 \%$, but it can still cause the large ionospheric disturbance.

Simulation 4 Heating the ionosphere during the solar maximum (2002) and solar minimum (2008). The ERP of transmitter is $200 \mathrm{MW}$, and radio frequency is $\sim \mathrm{foF}_{2}$. Figure 6 shows the disturbance of the electron temperature and density under the above heating conditions. At the solar maximum, the temperature disturbance of spring and autumn can reach $\sim 40 \%$ and $\sim 50 \%$ while the winter and summer are $\sim 20 \%$ and $\sim 30 \%$ respectively. At the solar minimum, the temperature disturbance of spring and autumn can reach $\sim 80 \%$ while the winter and summer are $\sim 65 \%$ and $\sim 50 \%$ respectively. The electron temperature disturbance in the solar minimum is larger than that in solar maximum and the disturbances in spring and autumn are larger than that in winter and summer.

\section{Conclusions}

Under the well-justified assumption of ambipolar diffusion, we solve the momentum equation, continuity equation and energy equation with Crank-Nicolson method, and calculate the change of electron temperature and density at different heating conditions. Main conclusions are shown as follows.

(1) Powerful HF radio waves can cause the electron temperature to increase markedly, and the largest increment is near the reflection layer. The electron density decreases at this area because of the grads of electron temperature makes the plasma diffuse along the magnetic field.

(2) The lower density of neutral background gas in the ionosphere, the larger disturbance amplitude of electron temperature and density; the higher radio frequency, the lower disturbance of electron temperature; and the higher radio power, the larger disturbance of electron temperature and density are caused, but there are no linear relationships between the radio power and disturbance amplitude.

(3) The disturbance at nighttime is larger than at daytime when heated by HF radio waves with the same powers. Possible reason is that the nighttime has lower plasma parameters than the daytime, when the radio wave with the same power interacts with ionosphere, more energy could transport to the electrons and induce larger ionospheric disturbance at nighttime.

(4) Compared with the low latitude, the mid-latitude has the smaller disturbances, the electron temperature is $\sim 30 \%$, and the density $\sim 5 \%$, but it can still cause large ionospheric disturbance.

(5) The electron temperature disturbance in the solar minimum is larger than that in solar maximum, and the spring and autumn are larger than that in winter and summer.

Simulation results show that the background neutral parameters have great influence on the heating results, but the outcomes from the empirical model IRI-2007 and NRLMISISE-00 are not the same as the true atmosphere parameters. Besides, the empirical equations are different from the theoretical equations. These make the differences between the quantitative calculations and experiment results, but have little effect on the qualitative conclusions in this paper.
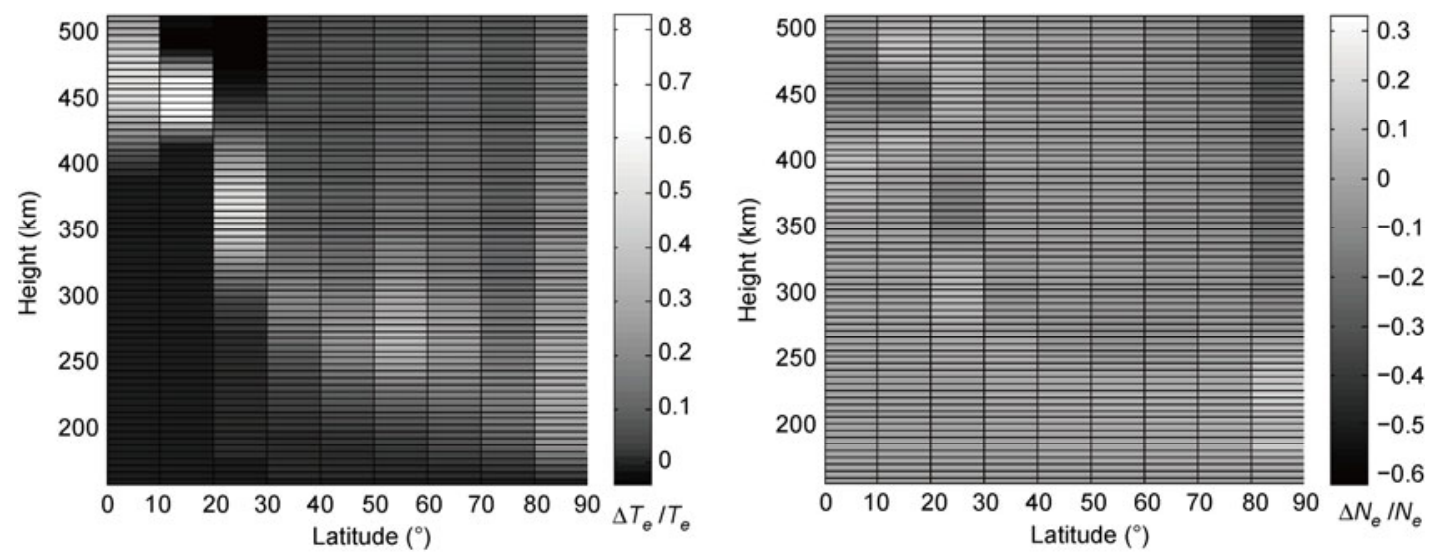

Figure 5 Profiles of electron temperature and density under the simulation 3 conditions. 

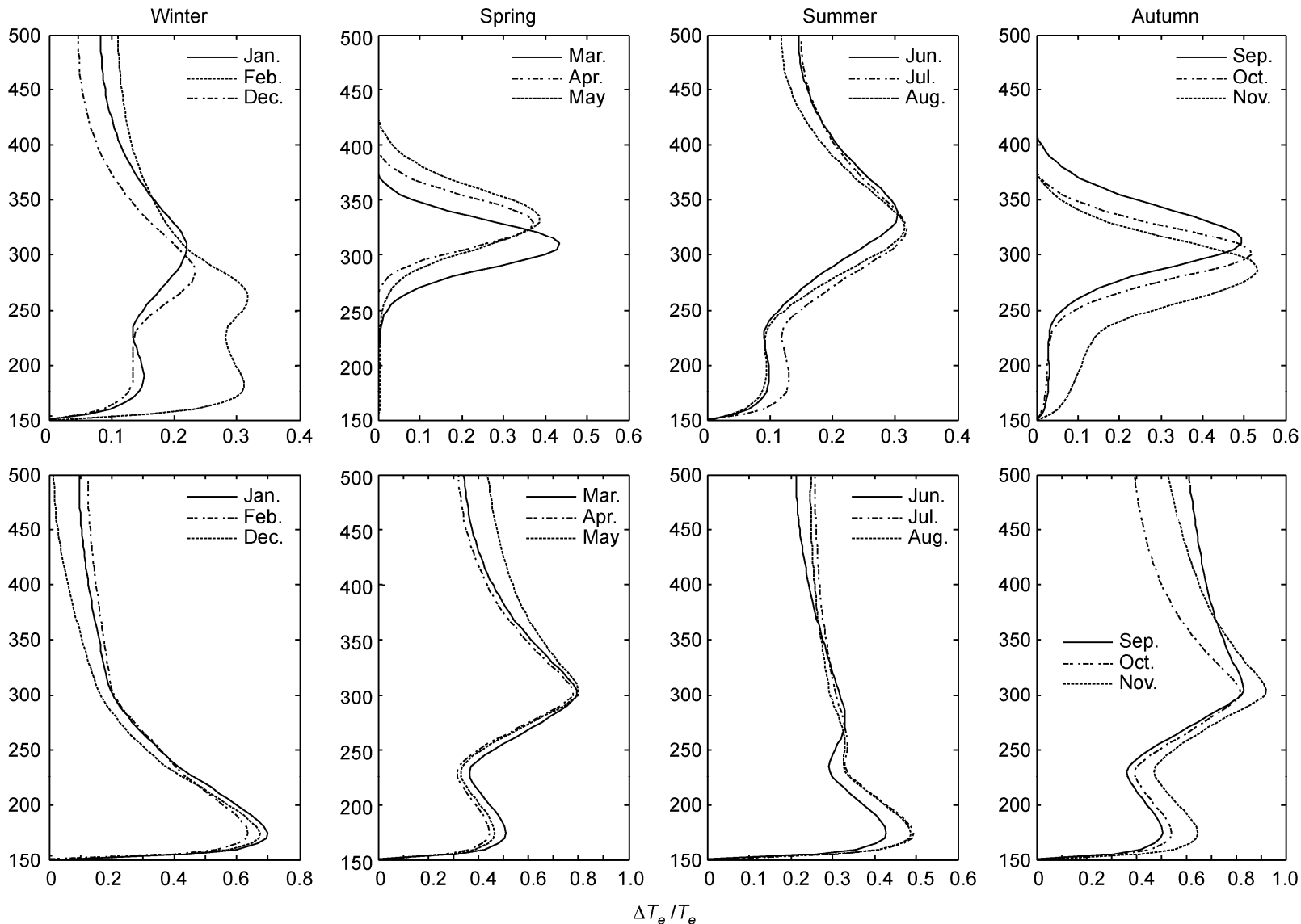

Figure 6 Profiles of electron temperature under the simulation 4 conditions. Upper panel is the solar maximum (2002) and the lower panel is maximum (2008).

The authors thank the URSI for providing IRI-2007 ionospheric model, the NRL for providing the model NRLMSISE-00. The authors also thank two anonymous referees for their help in evaluating this paper. This work was supported by the National Natural Science Foundation of China (40505005) and the Specialized Research Fund for State Key Laboratories.

1 Gorney D J. Solar cycle effects on the near-earth space environment. Rev Geophys, 1990, 28: 315-336

2 Forbes J M, Bruinsma S, Lemoine F G. Solar rotation effects in the thermospheres of Mars and Earth. Science, 2006, 312: 1366-1368

3 Liu L B, Wan W X, Chen Y D, et al. Solar activity effects of the ionosphere: A brief review. Chin Sci Bull, 2011, 56: 1202-1211

4 Abdu M A, Sobral J H A, de Paula E R, et al. Magnetospheric disturbance effects on the Equatorial Ionization Anomaly: An overview. J Atm Terr Phys, 1991, 53: 757-771

5 Astafyeva E I. Dayside ionospheric uplift during strong geomagnetic storms as detected by the CHAMP, SAC-C, TOPEX and Jason-1 satellites. Adv Space Rev, 2009, 43: 1749-1756

6 Inan U S, Bell T F, Rodriguez J V. Heating and ionization of the lower ionosphere by lightning. Geophys Res Lett, 1991, 18: 705-708

7 Nickolaenko A P, Hayakawa M. Heating of the lower ionosphere electrons by electromagnetic radiation of lightning discharges. $\mathrm{Ge}$ ophys Res Lett, 1995, 22: 3015-3018

8 Pulinets S A, Boyarchuk K. Ionospheric Precursors of Earthquakes. Berlin: Springer, 2004

9 Liu J Y, Chen Y I, Pulinets S A, et al. Seismo-ionospheric signatures prior to $M \geqslant 6.0$ Taiwan earthquakes. Geophys Res Lett, 2000, 27: 3113-3116

10 Le H, Liu J Y, Liu L. A statistical analysis of ionospheric anomalies before 763 M6.0+ earthquakes during 2002-2010. J Geophys Res, 2011, 116: A02303

11 Yao Y B, Chen $\mathrm{P}, \mathrm{Wu} \mathrm{H}$, et al. Analysis of ionospheric anomalies before the $2011 M_{\mathrm{w}} 9.0$ Japan earthquake. Chin Sci Bull, 2012, 57: 500-510

12 Yu T, Mao T, Wang Y G, et al. Study of the ionospheric anomaly before the Wenchuan earthquake. Chin Sci Bull, 2009, 54: 1080-1086

13 Mao T, Wang J S, Yang G L, et al. Effects of typhoon Matsa on ionospheric TEC. Chin Sci Bull, 2010, 55: 712-717

14 Lastovicka J. Effects of gravity and planetary waves on the lower ionosphere as obtained from radio wave absorption measurements. Phys Chem Earth C, 2001, 26: 381-386

15 Lastovicka J. Forcing of the ionosphere by waves from below. J Atmos Solar Terr Phys, 2006, 68: 479-497

16 Lastovicka J. Lower ionosphere response to external forcing: A brief review. Adv Space Res, 2009, 43: 1-14

17 Thide B. Artificial modification of the ionosphere: Preface. J Atmos Solar Terr Phys, 1997, 59: 2251-2252

18 Mendillo M, Forbes J M. Artificially created holes in the ionosphere. J Geophys Res, 1978, 83: 151-162

19 Li L, Xu R L. Neutral Barium cloud evolution at different altitudes. Chin Phys Lett, 2002, 19: 1214-1216

20 Willis S W, Davis J R. Radio frequency heating effect on the electron density in the lower E region. J Geophys Res, 1973, 78: 5710-5717

21 Gurevich A V. Nonlinear Phenomena in the Ionosphere. Berlin: Springer-Verlag, 1978

22 Bernhardt P A, Duncan L M. The feedback-diffraction theory of ionospheric heating. J Atmos Terr Phys, 1982, 44: 1061-1074

23 Shoucri M M, Morales G J, Maggs J E. Ohmic heating of the polar F 
region by HF pulses. J Geophys Res, 1984, 89: 2907-2917

24 Hansen J D, Morales G J, Maggs J E. Large scale HF-induced ionospheric modification: Theory and modeling. J Geophys Res, 1992, 97: 17019-17032

25 Huang W G, Gu S F. The heating of upper ionosphere by powerful high-frequency radio waves (in Chinese). Chin J Space Sci, 2003, 23: 343-351

26 Djuth F T, Sulzer M P, Elder J H. High resolutions observation of HF-induced plasma waves in the ionosphere. Geophys Res Lett, 1990, 17: $1893-1896$

27 Dubois D F, Rose H A, Russell D. Excitation of strong Langmuir turbulence in plasmas near critical density: Application to HF heating of the ionosphere. J Geophys Res, 1990, 21: 21221-21272

28 Banks P M, Kockarts G. Aeronomy: Part A and Part B. New York:
Academic Press, 1973

29 Belenov A F, Erukhimov L M, Ponomarenko P V, et al. Interaction between artificial ionospheric turbulence and geomagnetic pulsations. J Atmos Terr Phys, 1997, 59: 2367-2372

30 Stubbe P, Varnum W S. Electron energy transfer rates in the ionosphere. Planet Space Sci, 1972, 20: 121-126

31 Fu Z F, Hu Y Q. Numerical Simulations of Space Plasma (in Chinese). Hefei: Anhui Science and Technology Press, 1995

32 Hansen J D, Morales G J, Duncan L M, et al. Large-scale HF-induced ionospheric modification: Experiments. J Geophys Res, 1992, 97: 113-122

33 Duncan L M, Sheerin J P, Behnke R A. Observation of ionospheric cavities generated by high-power radio waves. Phys Rev Lett, 1988, 61: 239-242

Open Access This article is distributed under the terms of the Creative Commons Attribution License which permits any use, distribution, and reproduction in any medium, provided the original author(s) and source are credited. 